\section{Patienten mit Hirnfiliae: nicht übertherapieren!}

\section{Es gibt Hinweise und Daten, wonach das Überleben von Patienten mit Hirnmetastasen und schlechten Prognosefaktoren durch eine optimale Supportivtherapie anstelle einer Strahlentherapie nicht verkürzt wird.}

$\mathrm{D}$ urch die Entwicklung prognostischer Scores versucht man seit Jahren, Orientierungshilfen zu schaffen, um die Wahl der für den Einzelpatienten am besten geeigneten Therapie zu erleichtern. Da die tatsächlichen Überlebenszeiten stark streuen, ist eine Entscheidung für oder gegen den Einsatz einer palliativen Strahlentherapie beim einzelnen Patienten mit ungünstigen Prognosefaktoren weder auf der Basis vorhandener Instrumente wie des GPA (Graded Prognostic Assessment)-Index noch einer Kombination verschiedener Scores möglich.

Norwegische Autoren suchten nach zuverlässigeren Prädiktoren. In einer retrospektiven monozentrischen Studie wurden die Verläufe konsekutiver Patienten mit schlechten Prognose-Merkmalen (0 bis 1,5 Punkte im allgemeinen bzw. im Primärtumor-spezifischen GPA-Score) ausgewertet und die Überlebenszeiten verglichen. Die 124 Patienten hatten entweder Best Supportive Care (BSC), Ganzhirnbestrahlung (WBRT) oder eine stereotaktische Radiotherapie bekommen.

Allein anhand des Diagnose-spezifischen Scores konnte nach den Ergebnissen nicht ausreichend sicher abgeschätzt werden, ob eine Strahlentherapie einem bestimmten Patienten Nutzen bringt. Die Autoren fanden jedoch eine Subgruppe von 63 Patienten $(51 \%$ der Stichprobe), die unabhängig vom gewählten Vorgehen nur kurze Zeit überlebten. Diese Gruppe wies zusätzlich zu dem schlechten Score-Wert eines der folgenden Charakteristika auf:

- Alter mindestens 75 Jahre oder

- Karnofsky-Index $\leq 50$ oder

-unkontrollierter Primärtumor mit extrakraniellen Metastasen in mehr als einem Organ.

Angesichts fehlender Unterschiede beim Überleben zwischen den Studienarmen ist zu schließen, dass bei diesen Patienten der Verzicht auf eine Bestrahlung nicht zu Lasten der Überlebenszeit geht.

Fazit: BSC ist eine vernünftige Wahl bei begrenzter Lebenserwartung. Sollten die vorgestellten Selektionskriterien in weiteren Untersuchungen validiert werden, ließe sich die Entscheidung für oder gegen eine weitere Therapie auf eine solidere Basis stellen. Waldtraud Paukstadt

Nieder $C$ et al. Best supportive care in patients with brain metastases and adverse prognostic factors: development of improved decision aids. Support Care Cancer. 2013 May 18. [Epub ahead of print]

\section{Neutropenie und Fieber - Möglichkeiten und Grenzen der Prognoseabschätzung}

\begin{abstract}
Patienten mit febriler Neutropenie sind eine sehr heterogene Population in Bezug auf Schwere der möglichen Komplikationen und Prognose. Mit dem MASCC-Score lassen sich Patienten, bei denen mit hoher Wahrscheinlichkeit kein fulminanter Infektionsverlauf zu erwarten ist, herausfiltern.
\end{abstract}

$\mathrm{D}$ er validierte Risiko-Score der Multinational Association of Supportive Care in Cancer (MASCC) berücksichtigt mit unterschiedlicher Gewichtung Faktoren wie Krankheitslast, Vorhandensein von Hypotonie, COPD, Alter etc. (Tab. 1). Er hat sich inzwischen auch bei Hämatologie-Patienten als wertvolles Instrument erwiesen, Patienten mit niedrigem Komplikationsrisiko herauszufiltern. In jüngeren Studien wurde ihm eine hohe Sensitivität bei guter Spezifität bescheinigt. Betroffene mit niedrigem Risiko können sicher mit oralen Antibiotika behandelt und frühzeitig aus der Klinik entlassen werden. Orales Moxifloxacin 1-mal täglich ist bei ihnen offenbar so wirksam wie die Kombination aus Ciprofloxacin plus Amoxicillin/ Clavulansäure 2-mal täglich.
Durch die zuverlässige Identifizierung von Low-risk-Patienten, die nach einfacherem Protokoll therapiert werden können, ist die Implementierung eines Behandlungspfads möglich, mit dem die stationäre Gesamtmortalität gesenkt und die Dauer der Antibiotika-Behandlung verkürzt werden kann. Ein ambulantes Management sorgfältig ausgewählter Patienten ist genauso sicher und effektiv wie eine Standardtherapie in der Klinik - und kostengünstiger.

Grundsätzlich ist jedoch jede febrile Neutropenie ein Notfall. Besondere Eile ist bei höherem Risiko, d.h. niedrigem MASCC-Score geboten.

Fazit: Der MASCC-Score eignet sich nicht dazu, ein sehr hohes Komplikationsund/oder Mortalitätsrisiko vorherzusa-

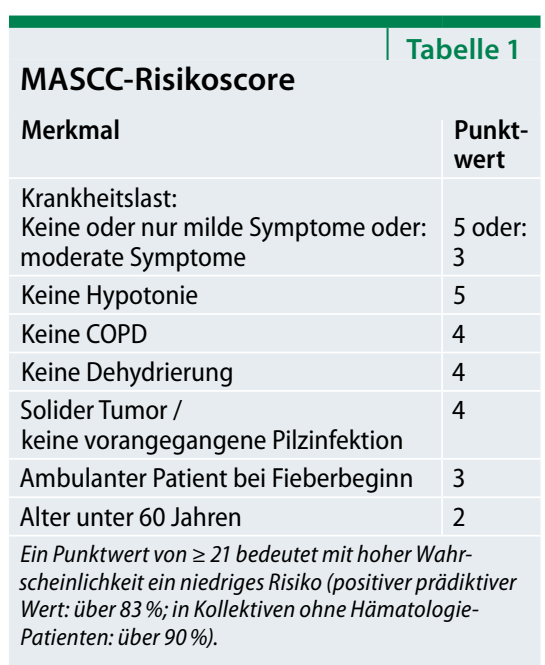

gen. Hochrisikopatienten rechtzeitig zu erkennen, bleibt weiterhin eine Herausforderung: Sie müssen umgehend, d.h. innerhalb eines Fensters von einer Stunde behandelt werden. Waldtraud Paukstadt

Klastersky J et al. The Multinational Association for Supportive Care in Cancer (MASCC) risk index score: 10 years of use for identifying low-risk febrile neutropenic cancer patients. Support Care Cancer. 2013;21(5):1487-95. 